\title{
Competencias investigativas en docentes universitarios. El caso del departamento de arquitectura de la Universidad Francisco de Paula Santander
}

\author{
Investigative competences in university professors. The case of the architecture \\ department of the Francisco de Paula Santander University \\ Competências de investigação em professores universitários. O caso do \\ departamento de arquitetura da Universidade Francisco de Paula Santander \\ Erika Tatiana Ayala Garcia*a , Jennyfer Magerly Barrera Prieto. ${ }^{\text {b }}$ \\ ${ }^{a}$ Magister en Estudios Territoriales y de la Población, Universidad Francisco de \\ Paula Santander.(Cúcuta, Colombia); (D) 'bagíster en Arquitectura, Universidad de \\ Ibagué, (Cúcuta-Colombia).
}

Forma de citar: Ayala, E. \& Barrera, J. (2018). Competencias investigativas en docentes universitarios. El caso del departamento de arquitectura de la Universidad Francisco de Paula Santander. Perspectivas, 3(1). 71-84

Recibido: septiembre 05 de 2017

Aceptado: diciembre 22 de 2017

\section{Palabras clave \\ Competencia \\ investigativa, docente, educación superior.}

\begin{abstract}
Resumen: El presente artículo permite determinar las competencias investigativas que poseen los docentes universitarios adscritos al departamento de Arquitectura, diseño y urbanismo de la Universidad Francisco de Paula Santander. Dentro de esta investigación se utilizó un enfoque cuantitativo y descriptivo de campo, a través de herramientas como la encuesta y la estadística descriptiva. La implementación de este enfoque favoreció el análisis de datos sobre las competencias investigativas en los docentes, partiendo de su propia autoevaluación dentro de una muestra compuesta por 25 docentes. En los resultados se identifican y caracterizan las competencias que poseen los docentes universitarios en materia de investigación. Así mismo, se proponen soluciones a los problemas detectados, por medio de conceptos y estrategias en base a las competencias investigativas, que contribuyan en el desarrollo y mejoramiento profesional de los docentes.
\end{abstract}

\footnotetext{
${ }^{1}$ El presente artículo se encuentra inscrito dentro de las investigaciones del grupo TARGET y se deriva de la tesis denominada "Competencias investigativas en docentes de educacion superior. El caso del programa de arquitectura de la universidad francisco de paula santander" para la especializacion en Educación, emprendimiento y economia solidaria de la UFPS. Tesis realizada en el marco del macro proyecto "Productividad científica: actitudes para la generación y divulgación del conocimiento en la educación superior" del grupo de investigacion GIPPEP.

* Autor para correspondencia

erikatatianaayala@ufps.edu.co
}

https://doi.org/10.22463/25909215.1425 


\section{Keywords}

Investigative competence, teaching, higher education.

\section{Palavras chave \\ Competência de inves- tigação, ensino, ensino superior.}

\begin{abstract}
The present article allows to determine the investigative competences that the university professors have assigned to the department of Architecture, design and urbanism of the Francisco de Paula Santander University. Within this research, a quantitative and descriptive field approach was used, through tools such as the survey and descriptive statistics. The implementation of this approach favored the analysis of data on research competences in teachers, based on their own self-assessment within a sample composed of 25 teachers. In the results, the competences that the university professors possess in research matters are identified and characterized. Likewise, solutions are proposed to the detected problems, by means of concepts and strategies based on the investigative competences, that contribute in the development and professional improvement of the teachers.
\end{abstract}

Resumo: O presente artigo permite determinar as competências investigativas que os professores universitários atribuíram ao departamento de Arquitetura, design e urbanismo da Universidade Francisco de Paula Santander. Dentro desta pesquisa, foi utilizada uma abordagem de campo quantitativo e descritivo, através de ferramentas como a pesquisa e estatística descritiva. A implementação desta abordagem favoreceu a análise de dados sobre competências de pesquisa em professores, com base em sua própria auto-avaliação dentro de uma amostra composta por 25 professores. Nos resultados, as competências que os professores universitários possuem em questões de pesquisa são identificadas e caracterizadas. Do mesmo modo, são propostas soluções aos problemas detectados, por meio de conceitos e estratégias baseadas nas competências investigativas, que contribuem para o desenvolvimento e a melhoria profissional dos professores.

\section{Introducción}

La educación en el contexto nacional con el paso de los años ha presentado indudables cambios, que van de la mano de los procesos de investigación que se gestan dentro de sus unidades académicas. Por tal razón, se hace importante indagar dentro de las mismas, el nivel de competencias con las que cuentan los docentes. Esto teniendo en cuenta que para los docentes estas competencias favorecen la practicapráctica pedagógica, y el desarrollo de su quehacer profesional y disciplinar.
Dentro de las políticas establecidas por el Ministerio de Educación Nacional se contempla la importancia de la vinculación, el desarrollo y la producción de procesos investigativos dentro de la Educación Superior. A partir de este hecho, las universidades se presentan como el escenario perfecto para la producción y generación de nuevos conocimientos. Además, las mismas pueden promover la transmisión de las herramientas y procesos que hacen de los procesos investigativos en los estudiantes en formación, en aras de obtener 
una Colombia más participativa en términos de investigación e innovación.

Bajo este contexto, dentro de este artículo se determina el estado actual de las competencias investigativas que presentan los docentes adscritos al departamento de Arquitectura, diseño y urbanismo de la Universidad Francisco de Paula Santander. Esto con el ánimo de que sus resultados puedan llegar a ser tenidos en cuenta en el plan de mejoramiento desarrollado dentro del proceso de autoevaluación propio de la Acreditación en alta calidad, por medio de los factores de investigación $\mathrm{y}$ docentes.

\section{Las competencias en la educacion superior.}

En una primera instancia, vale la pena resaltar el pensamiento de autores como Castañeda Poveda, Diaz Barros, \& Florez Pardo(2015) quienes a traves del estudio de posturas de autores como Álvarez (2008), Tobón (2006), Salas (2002), Jaik \& Ortega (2013), Delgado(2006) y Pimienta (2012) aseguran que desde su origen el termino "competencia" ha presentado diferentes puntos de interpretación bajo la perspectiva sociologica, psicologica, pedagogica, lingüística que hace referencia al saber y al saber hacer, teniendo en cuenta la capacidad de la misma dentro de diferentes areas relacionadas con la ciencia de la educacion.

"Al analizar las diferentes concepciones de competencias en la educación, se puede inferir que no tienen contradicciones contundentes, varían eso sí, en las denominaciones que usan para describirlas. En términos generales, los autores antes mencionados, hablan de dos momentos de las competencias educativas. El primero, relacionado con la adquisición de información disciplinar básica. El segundo, referido a su empleo en la resolución de situaciones y problemas determinados. Estos dos momentos se relacionan con tres competencias generales: un saber (aspecto cognitivo), un saber hacer (uso del saber para solución de problemas) y un ser o saber ser (actitudes)". (p. 16)

Desde esta perspectiva, autores como Rodríguez Jimenez (2009) recuerdan que a partir de 1990 tras los planteamientos de Humboldt, se promueve la investigacion en el contexto educativo. Esto teniendo en cuenta que precisamente en Alemania de la mano de la Universidad de Berlin se genera un ambiente cientifico social a traves del cual se transmiten tanto creencias como valores y aptitudes; por medio de la cuales se incursiono en el desarrollo de investigaciones educacionales.

Este hecho favoreció la la reflexion en torno al significado de la educación con respecto a su papel en la construccion del conocimiento, teniendo en cuenta que desde el ambito pedagógico se constituye como un espacio de investigacion centrado en los diferentes procesos de aprendizaje y las direfentes situaciones desarrolladas dentro del contexto de las aulas de clase. (p. 26-28).

Ahora bien, con respecto a la investigación en la educación colombiana esta autora asegura que:

"De acuerdo con lo estipulado en la Ley 30 de 1992 (MEN, 1992) respecto a la investigación en la educación superior, ésta se refiere a la búsqueda y generación de conocimiento, a la experiencia de investigación de alto nivel. En este sentido, las políticas en educación superior abordan el tema de la investigación como eje fundamental en la construcción de conocimiento, como espacio para el diálogo y el desarrollo social.

Dentro de los planteamientos de la educación superior respecto a la investigación se encuentra una clara clasificación del mismo acorde con la forma y ámbito en que se desarrolló. La investigación formativa se constituye en pieza fundamental para la formación de profesionales y responde a varias inquietudes, como son la búsqueda de interrogantes, de problemas, hipótesis, poblaciones, para estructurar proyectos de investigación (...) 
La investigación formativa persigue el desarrollo de proyectos de investigación en el trabajo en comunidad, en donde la formación, propiamente dicha, se da en el ejercicio mismo de la investigación, esto es, en la práctica $(\ldots) "$. (p. 28)

Con base en esto, Murcia(2015) propone que en Colombia es importante otorgar una mayor valoracion a la investigacion dentro del ambito educativo, teniendo en cuenta que es precisamente mediante la misma que se puede fomentar el interes por el analisis y la reflexion tanto de los docentes como de los estudiantes por medio de enfoques de enseñanzas basados en la critica, la argumentacion $\mathrm{y}$ el analisis.

Además queda en evidencia que para los docentes, la puesta en práctica de la investigación dentro de su quehacer académico y profesional les permite reflexionar y profundizar sobre su práctica a partir de componentes de análisis, que sumados a las diversas metodologías y recursos favorecen procesos de autoevaluación tendientes al mejoramiento de su trabajo en el aula de clase.

Así las cosas, el papel que cumple la universidad dentro del mejoramiento de la calidad de vida del país, se encuentra directamente relacionado con la cantidad y calidad de recurso y talento humano que el mismo contenga, centrado en la capacidad de generar, transmitir conocimientos innovadores $\mathrm{y}$ creativos tendientes a satisfacer las necesidades del entorno en el que se desarrollan desde un perfil público y/o privado.

Para tal fin, las autoras Marrero \& Pérez(2014) mencionan que desde la labor se debe docente se impartir y fomentar la formación de competencias integrales investigativas que permitan una educación científica-cultural que responda a los ámbitos labores y personales en la que la investigación se presente como un eje transversal que permita promover competencias como el pensamiento crítico, el análisis y la síntesis; entre otros.

Desde esta perspectiva, Ochoa(2013) menciona que el docente debe vislumbrarse como el factor de mayor incidencia en la calidad de la educación, tomando para ello como punto de referencia la definición que maneja el ICFES con respecto al termino "competencia" entendiéndola como "la capacidad compleja que integra conocimientos, potencialidades, habilidades, destrezas, prácticas $\mathrm{y}$ acciones que se manifiestan en el desempeño en en situaciones concretas, en contextos específicos (saber hacer en forma pertinente).

Para tal fin, dentro del texto se describen los aspectos generales que componen las mismas, describiendo al docente como un ser humano comprometido tanto con su quehacer pedagógico como disciplinar que centra sus métodos de enseñanza en ámbitos como la comprensión y la apropiación del conocimiento por parte de los estudiantes. (p. 9-10)

Así mismo, el texto se destaca las competencias comunes e interconectadas con las que debe contar un docente, resaltando: Enseñar: comprender, formular y usar la didáctica de las disciplinas con el propósito de favorecer los aprendizajes, Formar: re conceptuar y utilizar conocimientos pedagógicos que permitan crear ambientes educativos para el desarrollo de los estudiantes y Evaluar: reflexionar, hacer seguimiento $\mathrm{y}$ tomar decisiones sobre los procesos de formación, con el propósito de favorecer la autorregulación y de plantear acciones de mejora en la enseñanza, en el aprendizaje y en el currículo. (p. 11).

Está claro que, a partir de estos lineamientos, los docentes de educación superior deben centrar sus prácticas en el fomento de competencias encaminadas al fortalecimiento de la formación, comprensión y el compromiso, por medio de estratégicas pedagógicas que favorezcan el aprovechamiento de la innovación en beneficio de las problemáticas de la sociedad donde se encuentran.(Marrero \& Pérez, 2014)

Desde esta perspectiva, Salazar, Chiang \& Muñoz(2016) a traves del estudio de autores como Segura(2004), Quinn, Faerman, Thompson, \& McGrath (1994) y Corvalán(2009) aseguran que los docentes de educación superior deben contar 
dentro de su práctica pedagógica con perfiles que respondan a la capacidad de adaptación de los diferentes contextos y circunstancias sociales en donde se desenvuelven promoviendo en los estudiantes la innovación a través del liderazgo y la comprensión propia de la educación.

En base a todo lo anterior, dentro del estudio desarrollado por Aular de Durán, Marcano, \& Moronta(2009) las competencias investigativas se definen como la capacidad que tienen los docentes de interpretar, argumentar, proponer, preguntar y escribir sobre la experiencia pedagógica con respecto a una problemática específica, por medio de un argumento critico acompañado de un desempeño académico eficiente que fomente el intercambio y la confrontación de ideas.

Así las cosas, autores como Muñoz \& Quintero(2001) establecen que el propósito del desarrollo de las competencias investigativas se encuentra encaminado básicamente a: Comprender el significado, la importancia y las implicaciones de la investigación educativa en la práctica pedagógica del educador, observar, preguntar, registrar, interpretar, analizar, describir contextos y escribir textos acerca de situaciones problemáticas propias de los ambientes escolares, proponer soluciones a los problemas detectados, utilizando los conceptos y los métodos de investigación, sean éstos explicativos, interpretativos o críticos.

De la misma manera a argumentar sobre las relaciones que se establecen dentro de la cultura escolar y las alternativas que se dan a los problemas investigativos detectados, perfeccionar las prácticas escriturales, redactando notas de campo sintetizando datos, textos y sistematizando informes de investigación acerca de un problema identificado en el aula o en la escuela.(p.146)

En base en esto, dentro de esta investigación se plantea que el desarrollo de competencias dentro de programas como la arquitectura son importantes debido a que:

"La necesidad de desarrollar competencias investigativas, con el fin de formar en este caso ingenieros y arquitectos comprometidos, en pro de generar procesos dinámicos y continuos de aprendizaje social para la producción y transmisión de conocimientos y tecnologías $y$ que en su forma de actuación establezcan valores y actitudes que les permitan asumir, suscitar e impulsar ideas y cambios acordes con el momento histórico; (...) también es necesario integrar la investigación con la producción de bienes y servicios, fomentar la actitud de liderazgo, para que se realicen proyectos sociales encaminados al desarrollo económico sostenible, generar alianzas estratégicas entre el sector productivo y la sociedad, como vía para el intercambio y la colaboración en la solución de problemas de interés social". (Nápoles, Beatón, Cruz, \& Álvarez, 2007, p. 11)

Por tal razón, la idea de que los docentes reconozcan y utilicen dentro de sus prácticas pedagógicas y quehaceres disciplinares la investigación favorece nuevos escenarios de formación encaminados al entendimiento de contextos globalizados, en donde los individuos tienen la capacidad de ser autónomos.

\section{Recursos, Materiales y Método.}

Esta investigación identificó las competencias investigativas que poseen los docentes los adscritos al departamento de Arquitectura de la Universidad Francisco de Paula Santander, Cúcuta. Las actividades de este estudio se basan en la metodología cuantitativa bajo un proceso no experimental-descriptivo, transversal-de campo, el cual permite averiguar las condiciones bajo las cuales se presenta un fenómeno .

En primer lugar, se debe definió el problema del estudio para poder establecer el tipo y diseño de investigación a llevar a cabo. El siguiente paso consistió en definir la muestra, efectuando la recolección y el análisis de los datos que se obtuvieron por medio de la investigación. Este 
estudio se realizó el Departamento de Arquitectura Diseño y Urbanismo de la Universidad Francisco de Paula Santander - Cúcuta. Sólo se estudió al personal docente para poder determinar las competencias investigativas de los docentes de los niveles de educación superior.

Esta investigación se realizó a partir de un estudio descriptivo de enfoque cuantitativo, pues recolecta datos o componentes sobre diferentes aspectos del personal de la organización a estudiar, realizando a partir de los mismos un análisis y una medición. "La investigación descriptiva busca especificar propiedades, características y rasgos importantes de cualquier fenómeno que se analice" (Hernández, Fernández, \& Baptista, 2003,pp. 119). Así mismo, el estudio tiene un enfoque cuantitativo, necesario para poder analizar los resultados de las encuestas aplicadas a los docentes objeto de estudio.

La población se centra en los docentes que prestan sus servicios al Departamento Arquitectura, diseño y urbanismo, integrada por 25 docentes que impartieron clases durante el II semestre de 2016 y el I semestre de 2017 en todas las asignaturas correspondientes al programa de Arquitectura de la Universidad Francisco de Paula Santander.

Se utilizó un muestreo probabilístico aleatorio simple, para determinar el tamaño de la muestra se utilizó un margen de confianza del 95\% y 5\% de error (Martínez, 2012). En total la muestra fue conformada por 25 docentes.

La técnica de recolección de información utilizada fue la encuesta. A través de la encuesta se buscó obtener información cuantitativa de los datos a medir, correspondientes a las competencias investigativas de los docentes. Esta herramienta de recolección de investigación abarcóen primer lugar una escala tipo Likert y en segundo lugar los datos del perfil socioeducativas de los docentes.

Con el diseño de este instrumento, en la investigación se pudo observar cuál es el impacto de las competencias investigativas del docente en los procesos generación y divulgación del conocimiento en la Educación Superior.

Los datos fueron analizados a través de técnicas descriptivas de correlación y análisis de varianza, se adoptó el uso del software estadístico IBM SPSS Statistics 22 y la hoja de cálculo Microsoft Excel para el proceso de análisis de los datos.

\section{Resultados y Analisis.}

Los resultados de este estudio buscan responder a la pregunta de investigación ¿Cuáles son las competencias investigativas que poseen los docentes del departamento de Arquitectura, Diseño y Urbanismo de la Universidad Francisco de Paula Santander?, mediante categorías basescomo: Niveles de competencias, Dimensión de identificación y organización de la información, Dimensión de generación científica del conocimiento, Dimensión de divulgación de conocimientos e información investigativa de los docentes.

Niveles de Competencias. La encuesta aplicada a los docentes del Departamento de Arquitectura Diseño y Urbanismo del Universidad Francisco de Paula Santander, Cúcuta está conformada por 3 categorías: Primera Categoría: Identificación y Organización de la Información compuesta por 12 ítems, segunda Categoría: Generación Científica del Conocimiento compuesta por 14 ítems, tercera Categoría: Divulgación de Conocimientos por 9 ítems.

Una vez realizada su aplicación se efectúo la identificación de los datos y la generación de los promedios o porcentajes.

${ }^{2}$ Se resalta que el enfoque de investigación utilizado en este proyecto, corresponde al implementado en el macro proyecto del cual se deriva denominado

"Productividad científica: actitudes para la generación y divulgación del conocimiento en la educación superior". 
Tabla 1.

Resultados de las dimensiones de las competencias.

\begin{tabular}{lccc}
\hline Dimensiones de las Competencias & Promedio & Ítems & $\begin{array}{c}\text { Desviación } \\
\text { Estándar }\end{array}$ \\
\hline $\begin{array}{l}\text { Identificación y Organización de } \\
\text { la Información }\end{array}$ & 4.45 & 12 & 0.76 \\
\hline $\begin{array}{l}\text { Generación Científica del } \\
\text { Conocimiento }\end{array}$ & 4.41 & 14 & 0.68 \\
\hline Divulgación de Conocimientos & 4.07 & 9 & 0.92
\end{tabular}

En esta tabla 1 se puede evidenciar que dentro de las dimensiones de las competencias la Identificación y Organización de la Información presenta el valor medio más alto de 4,45, seguida por Generación Científica del Conocimiento $(4,41)$ y la Divulgación de Conocimientos (4,07). También se puede observar que la distancia entre la Generación Científica del Conocimiento y la Identificación y Organización de la Información es muy mínima. un nivel de competente, posiblemente influenciado porque la mayoría de ellos cuentan con formación pos gradual.

Dimensión de Identificación y Organización de la Información. En la siguiente tabla presentan de manera condensada los indicadores de las competencias para la Identificación y Organización de la Información. De acuerdo con lo anterior, los docentes exhiben

Tabla 2.

Resultados de indicadores: Identificación y Organización de la Información.

\begin{tabular}{|c|c|c|}
\hline Indicador & Media & $\begin{array}{l}\text { Desviación } \\
\text { Estándar }\end{array}$ \\
\hline Identifico fuentes de información que facilitan mis procesos de investigación. & 4,60 & 0,60 \\
\hline $\begin{array}{l}\text { Identifico bases de datos científicas y académicas para soportar mis procesos de } \\
\text { investigación }\end{array}$ & 4,60 & 0,58 \\
\hline $\begin{array}{l}\text { Sé distinguir entre publicaciones científicas de prestigio (arbitradas e indexadas) y } \\
\text { publicaciones no científicas }\end{array}$ & 4,60 & 0,76 \\
\hline Ubico con facilidad información valida y confiable disponible en internet & 4,63 & 0,82 \\
\hline Utilizo la información disponible en Internet con una actitud crítica y reflexiva & 4,68 & 0,63 \\
\hline $\begin{array}{l}\text { Utilizo plataformas especializadas (repositorios, bases de datos, software } \\
\text { científico) para sustentar mis investigaciones }\end{array}$ & 4,32 & 0,99 \\
\hline Ordeno los resultados de la búsqueda de acuerdo con el interés de la investigación & 4,20 & 0,91 \\
\hline Selecciono las fuentes de acuerdo con su relevancia y afinidad para el estudio & 4,60 & 0,65 \\
\hline Evito concatenar citas sin comentarlas o relacionarlas entre sí & 4,04 & 1,00 \\
\hline $\begin{array}{l}\text { Estoy en capacidad de exponer sintética y ordenadamente el conocimiento } \\
\text { previamente publicado relacionado con la investigación }\end{array}$ & 4,44 & 0,71 \\
\hline $\begin{array}{l}\text { Extraigo las ideas originales de otros autores y las incorporo apropiadamente para } \\
\text { soportar mis argumentos }\end{array}$ & 4,52 & 0,59 \\
\hline Procuro en la medida de lo posible usar sólo fuentes primarias & 4,20 & 0,91 \\
\hline
\end{tabular}


En esta tabla 2 se puede evidenciar claramente como "Evito concatenar citas sin comentarlas o relacionarlas entre sí", "Ordeno los resultados de la búsqueda de acuerdo con el interés de la investigación", "Procuro en la medida de lo posible usar sólo fuentes primarias", y "Utilizo plataformas especializadas (repositorios, bases de datos, software científico) para sustentar mis investigaciones", son los indicadores que presentaron menor promedio entre los 12 ítems de esta competencia.

Este hecho demuestra que los docentes sometidos a estudio manifiestan un porcentaje bajo en categorías como: métodos de análisis de datos, componer y redactar informe de investigación, selección de participantes de estudio, adecuar el formato de resultado de la investigación en los sitios que se presentan.

\section{Dimensión de Generación Científica} del Conocimiento. A continuación, se muestra de manera condensada los indicadores de las competencias para la Generación Científica del Conocimiento:

Tabla 3.

Resultados de indicadores: Generación Científica del Conocimiento.

\begin{tabular}{|c|c|c|}
\hline Indicador & Media & $\begin{array}{l}\text { Desviación } \\
\text { Estándar }\end{array}$ \\
\hline $\begin{array}{l}\text { Reconozco en la realidad circundante situaciones o problemas que pueden ser } \\
\text { objeto relevante de una investigación }\end{array}$ & 4,58 & 0,78 \\
\hline $\begin{array}{l}\text { Tengo la capacidad para formular adecuadamente como problema de } \\
\text { investigación una situación de la realidad }\end{array}$ & 4,40 & 0,82 \\
\hline $\begin{array}{l}\text { Tengo claro que toda investigación debe realizar una aportación original y } \\
\text { relevante a un área específica del conocimiento }\end{array}$ & 4,68 & 0,56 \\
\hline $\begin{array}{l}\text { Puedo formular en términos de hipótesis o preguntas de investigación un } \\
\text { problema científico }\end{array}$ & 4,52 & 0,59 \\
\hline Sé fundamentar ideas de otros autores para construir un argumento & 4,48 & 0,51 \\
\hline Puedo formular en términos claros y precisos el propósito de una investigación & 4,60 & 0,58 \\
\hline $\begin{array}{l}\text { Distingo entre las metodologías de investigación la más adecuada a un problema } \\
\text { determinado }\end{array}$ & 4,36 & 0,70 \\
\hline $\begin{array}{l}\text { Selecciono los participantes del estudio a través de procedimientos cónsonos con } \\
\text { las metodologías empleadas }\end{array}$ & 4,25 & 0,79 \\
\hline $\begin{array}{l}\text { Reconozco el tipo de instrumento de recolección de datos adecuado a la } \\
\text { naturaleza y propósito de la investigación }\end{array}$ & 4,48 & 0,71 \\
\hline $\begin{array}{l}\text { Conozco herramientas básicas de análisis estadístico para el tratamiento de los } \\
\text { resultados obtenidos en investigaciones }\end{array}$ & 3,96 & 0,86 \\
\hline $\begin{array}{l}\text { Tengo capacidad para elaborar tablas o gráficos que resumen el producto de mis } \\
\text { investigaciones }\end{array}$ & 4,38 & 0,77 \\
\hline Conozco métodos para analizar datos de naturaleza no numéricas & 4,08 & 0,76 \\
\hline $\begin{array}{l}\text { Estoy en capacidad de contrastar los resultados obtenidos con los de otras } \\
\text { investigaciones y posiciones teóricas }\end{array}$ & 4,36 & 0,64 \\
\hline $\begin{array}{l}\text { Conozco los elementos éticos que deben seguirse en las investigaciones en las } \\
\text { que participan seres humanos }\end{array}$ & 4,60 & 0,50 \\
\hline
\end{tabular}


En esta tabla 3 se puede evidenciar claramente como "Conozco herramientas básicas de análisis estadístico para el tratamiento de los resultados obtenidos en investigaciones", "Conozco métodos para analizar datos de naturaleza no numéricas", y en "Selecciono los participantes del estudio a través de procedimientos cónsonos con las metodologías empleadas", son los indicadores que presentaron menor promedio entre los 14 ítems de esta competencia. Este hecho deja en evidencia que los docentes del Departamento de Arquitectura, diseño y urbanismo de la UFPS, Cúcuta tienen un porcentaje alto en cuanto a Generación Científica del Conocimiento.

\section{Dimensión de Divulgación de Conocimientos.}

En la siguiente tabla muestra de manera condensada los indicadores de las competencias para la Divulgación de Conocimientos:

Tabla 4.

Resultados de indicadores: Divulgación de Conocimientos.

\begin{tabular}{|c|c|c|}
\hline Indicador & Media & $\begin{array}{l}\text { Desviación } \\
\text { Estándar }\end{array}$ \\
\hline $\begin{array}{l}\text { Conozco los distintos escenarios (reuniones científicas y publicaciones) para la divulgación } \\
\text { de la investigación }\end{array}$ & 4,38 & 0,77 \\
\hline $\begin{array}{l}\text { Distingo la necesidad de adecuar el formato de los resultados de la investigación al espacio en } \\
\text { el que se presentan (blogs, artículos científicos, ponencias y redes sociales) }\end{array}$ & 4,48 & 0,65 \\
\hline $\begin{array}{l}\text { Participo en eventos (foros, seminarios, congresos) con el fin de divulgar los resultados de } \\
\text { mis investigaciones }\end{array}$ & 3,92 & 1,26 \\
\hline Reconozco la importancia de normas estandarizadas para las publicaciones científicas & 4,48 & 0,65 \\
\hline Tengo capacidad para componer y redactar un informe de investigación en forma adecuada & 4,32 & 0,63 \\
\hline $\begin{array}{l}\text { Participo en redes, basadas en el uso de tecnologías, para la construcción colectiva de } \\
\text { conocimiento. }\end{array}$ & 3,88 & 0,90 \\
\hline $\begin{array}{l}\text { Participo en comunidades virtuales, para la construcción colectiva de conocimiento con el } \\
\text { apoyo de TIC. }\end{array}$ & 3,67 & 1,13 \\
\hline $\begin{array}{l}\text { Participo en proyectos colaborativos, a través del uso de TIC, para la construcción colectiva } \\
\text { de conocimiento. }\end{array}$ & 3,58 & 1,21 \\
\hline $\begin{array}{l}\text { Selecciono apropiadamente el medio de divulgación de una investigación de acuerdo con la } \\
\text { pertinencia de los resultados obtenidos a la línea editorial }\end{array}$ & 3,92 & 1,06 \\
\hline
\end{tabular}

En esta tabla 4 se puede evidenciar que los ítems "Distingo la necesidad de adecuar el formato de los resultados de la investigación al espacio en el que se presentan (blogs, artículos científicos, ponencias y redes sociales)" y "Reconozco la importancia de normas estandarizadas para las publicaciones científicas" fueron los indicadores que presentaron mayor promedio. Esto demuestra en términos generales los docentes tienen un porcentaje bajo en cuanto a la participación en eventos (charlas, seminarios, congresos) con el fin de divulgar los resultados de sus investigaciones. 
Información Investigativa de los docentes. A Departamento de Arquitectura Diseño y Urbanismo continuación, se muestra de manera condensada las de la UFPS. características socio académicas de los docentes del

Tabla 5.

Características socio académicas de los docentes encuestados.

\begin{tabular}{|c|c|c|}
\hline VARIABLE & ESCALA & $\%$ \\
\hline \multirow[t]{2}{*}{ Género } & Femenino & 44,0 \\
\hline & Masculino & 56,0 \\
\hline \multirow[t]{2}{*}{ Edad } & Promedio & 38,2 \\
\hline & Desviación estándar & 7,0 \\
\hline \multirow[t]{3}{*}{ Tipo de contratación } & Catedra & 76,0 \\
\hline & Ocasional & 8.0 \\
\hline & Planta & 16,0 \\
\hline \multirow[t]{4}{*}{ Escalafón docente } & Auxiliar & 42,9 \\
\hline & Asociado & 23,8 \\
\hline & Asistente & 23,8 \\
\hline & Titular & 9,5 \\
\hline \multirow[t]{2}{*}{ Años de experiencia en docencia universitaria } & Promedio & 6,6 \\
\hline & Desviación estándar & 4,8 \\
\hline \multirow[t]{2}{*}{ Años de experiencia en investigación } & Promedio & 5,1 \\
\hline & Desviación estándar & 5,7 \\
\hline \multirow[t]{3}{*}{ Nivel posgradual } & Especialización & 37,5 \\
\hline & Maestría & 56,3 \\
\hline & Doctorado & 6,3 \\
\hline
\end{tabular}

En cuanto a la distribución de los docentes encuestados en función del género se puede observar que por cada 10 docentes aproximadamente 6 de ellos son de género masculino. El promedio de edad es de 38,2 con una desviación estándar de 7,0. Lo que muestra la necesidad de incorporar nuevos docentes con menor edad, pero mayor preparación académica. 
El tipo de contratación muestra que el 76,0\% de los docentes encuestados que laboran en el Departamento de Arquitectura Diseño y Urbanismo posee un contrato de cátedra, por el contrario, menos del $16,0 \%$ son docentes de planta. Este hecho resalta la necesidad de vincular más docentes de planta, preferiblemente con formación en Investigación.

La mayoría de los docentes encuestados se ubican en los 2 primeros niveles del escalafón (auxiliar y asistente con un 42,9 y 23,8\% respectivamente cada uno). El promedio de años de experiencia en docencia universitaria es de 6,6 con una desviación estándar de 4,8, pero se puede observar que el promedio en años de experiencia investigativa es de 5,1 con una desviación estándar de 5,7. Estos resultado reflejan los docentes del Departamento de Arquitectura Diseño y Urbanismo deben adquirir experiencia investigativa y que por lo tanto deben formarse en investigación.

El nivel de maestría constituye el nivel académico que se indica con mayor frecuencia por los docentes encuestados, seguido del nivel académico de especialización. Lo que muestra que la gran mayoría de los docentes tiene un nivel de formación alto en el Departamento de Arquitectura Diseño y Urbanismo, pero baja formación en investigación.

Tabla 6.

Diagnostico general relacionado con los procesos investigativos que hace los docentes del Departamento de Arquitectura Diseño y Urbanismo de la UFPS.

\begin{tabular}{|c|c|c|}
\hline Ítems & SI & NO \\
\hline $\begin{array}{l}\text { Ha recibido formación en } \\
\text { investigación }\end{array}$ & 72,0 & 28,0 \\
\hline $\begin{array}{l}\text { Pertenece algún grupo de } \\
\text { investigación }\end{array}$ & 48,0 & 52,0 \\
\hline Tiene CVLAC & 68,0 & 32,0 \\
\hline $\begin{array}{l}\text { Ha participado en proyectos de } \\
\text { investigación }\end{array}$ & 75,0 & 25,0 \\
\hline $\begin{array}{l}\text { Ha participado en proyectos de } \\
\text { investigación financiado }\end{array}$ & 45,8 & 54,2 \\
\hline $\begin{array}{l}\text { Los resultados de estas } \\
\text { investigaciones han sido } \\
\text { publicados }\end{array}$ & 78,9 & 21,1 \\
\hline $\begin{array}{l}\text { Ha realizado publicaciones no } \\
\text { indexadas }\end{array}$ & 63,6 & 36,4 \\
\hline $\begin{array}{l}\text { Ha realizado publicaciones } \\
\text { indexadas }\end{array}$ & 40,0 & 60,0 \\
\hline $\begin{array}{l}\text { Ha publicado revista de alto } \\
\text { impacto }\end{array}$ & 17,6 & 82,4 \\
\hline
\end{tabular}


En la tabla 6 se puede observar que menos de la mitad de los docentes del Departamento de Arquitectura Diseño y Urbanismo, manifestaron (con un 48\%) que pertenecen a algún grupo de investigación. Además, que el $75 \%$ ha participado en un proyecto de investigación, de los cuales el 45,8 financiado. Sin embargo, se resalta que en cuanto a la publicación de los resultados de sus investigaciones, los docentes han publicado en revistas no indexadas $(63,6 \%)$ y de bajo impacto $(82,4 \%)$.
De otro lado cerca de las tres cuartas partes de los docentes del Departamento de Arquitectura Diseño y Urbanismo, manifestaron con un $72,0 \%$, que sí han recibido formación investigativa a través de los cursos de posgrados; especialmente de las maestrías. De la misma manera, los docentes aseguraron contar con cursos de formación docente en investigación cuantitativa, cualitativa y redacción de artículos científicos. Además el $68.0 \%$ manifiestan que cuentan con CVLAC.

Tabla 7.

Herramientas que ha utilizado para realizar búsqueda de la información por parte de los docentes del Departamento de Arquitectura Diseño y Urbanismo.

\begin{tabular}{l}
\hline Bases de datos \\
\hline Google \\
\hline Google escolar \\
\hline Otras fuentes \\
\hline NS/NR \\
\hline
\end{tabular}

En tabla 7 se puede evidenciar claramente que las fuentes que generalmente usan los docentes del Departamento de Arquitectura Diseño y Urbanismo para sus investigaciones son las herramientas de Google: Google académico (28,0\%) y Google $(32,0 \%)$; destacando que en menor proporción se utilizan bases de datos (16\%).

\section{Conclusiones}

En base al instrumento aplicado a los docentes adscritos al departamento de Arquitectura, diseño y urbanismo de la Universidad Francisco de Paula Santander, se puede concluir que los docentes objeto de estudio manifiestan un nivel de competencia alto referente al tema de la investigación, coherente con su nivel de formación pos gradual.

Esto favorece considerablemente la práctica pedagógica de los mismos y trae como consecuencia que dentro de su práctica de docencia se puedan implementar procesos investigativos que complementen y enriquezcan la formación académica tanto de los estudiantes como de los mismos docentes.

Sin embargo, se debe resaltar que es claro que los docentes manifestaron bajos porcentajes referentes a: análisis de datos frente a los procesos investigativos, composición y redacción de informes de investigación, aspectos metodológicos como la selección de participantes de estudio; entre otros. Estos indicadores se presentan como una necesidad que debe estar contextualizada dentro del plan de mejoramiento del programa en los factores pertenecientes a la cualificación docente.

Lo anterior con el objetivo de que la institución brinde la oportunidad a los docentes de este departamento de adquirir las competencias necesarias con respecto a los procesos formales y metodológicos propios de la investigación.

De la misma manera, queda en evidencia que los docentes adscritos al departamento de arquitectura, diseño y urbanismo de la UFPS manifiestan un porcentaje bajo en cuanto a la participación en 
eventos (charlas, seminarios, congresos) con el fin de divulgar los resultados de sus investigaciones.

Este resultado se presenta a pesar de contar con dos grupos de investigación y dos semilleros como unidades investigativas adscritas al programa de Arquitectura. Se presume que esta condición pudiese llegar a estar concatenada al hecho de que el mayor porcentaje de los docentes del departamento se encuentran bajo la modalidad de cátedra, siendo los docentes de planta quienes asumen con mayor frecuencia esta actividad.

Así mismo, los docentes adscritos a este departamento requieren capacitación en lo referente al tema de las publicaciones, haciendo un énfasis especial en la importancia de reconocer y apostar por las revistas indexadas y reconocidas tanto nacional como internacionalmente. Además de utilizar los espacios de discusión de alto impacto con el fin de mejorar los indicadores referentes a la apropiación social del conocimiento y la producción de artículos; dentro de los estándares requeridos actualmente por Colciencias.

De igual manera, es necesario que se capacite a los docentes con respecto a la importancia de la utilización de las bases de datos como soporte de las investigaciones que realizan, teniendo como referencia la necesidad de contar con fuentes fidedignas y actualizadas.

Como punto general se establece que el escenario del departamento de arquitectura, diseño y urbanismo con respecto a las competencias investigativas de sus docentes adscritos se presenta como una oportunidad, teniendo en cuenta que los mismos manifiestan una base pos gradual que favorece el reconocimiento básico de los elementos propios de la investigación, así como el hecho de que gran parte de los mismos pertenecen a unidades investigativas.

Sin embargo, con el ánimo de fortalecer la implementación y el desarrollo de las competencias investigativas en la educación superior, y teniendo en cuenta la importancia que las mismas representan tanto para los docentes como los estudiantes (comunidad académica) se hace necesario que en esta dependencia se realicen ajustes y se capaciten a los docentes en materia de competencias investigativas.

Esto con el fin de que los mismos puedan aprovechar al máximo el escenario investigativo presente dentro de su departamento. Hecho que influirá en el mejoramiento de la generación y la discusión del conocimiento, así como la realización constante de la investigación dentro de las aulas de clase.

\section{Agradecimientos}

Se otorga un agradecimiento al Departamento de Arquitectura, diseño y urbanismo de la Universidad Francisco de Paula Santander, Cúcuta y a los docentes que participaron en el desarrollo de la encuesta previsto en el trabajo de campo de esta investigación.

\section{Referencias}

Álvarez Sáchez, Y. (2008). De las competencias de la educación superior a las competencias laborales. Gestión y sociedad, 109-125.

Aular de Durán, J., Marcano, N., \& Moronta, M. (2009). Competencias investigativas del docente en educación superior. Laurus, 15(30), 138-165. Castañeda Poveda, I., Diaz Barros, L. F., \& Florez Pardo, K. A. (2015). Estado de competencias investigativas de estudiantes de maestria en docencia e investigación universitaria, Universidad Sergio Arboleda, cohortes XII y XV. Bogotá. Universidad Sergio Arboleda.

Corvalán Vásquez, O. (2009). Calidad y evaluación de los aprendizajes y competencias en la Educación Superior. Revista Electrónica de Desarrollo de Competencias (REDEC), 1(3), 5-28.

Delgado Garcia, A. M. (2006). competencias y diseño de la evaluación continua y final en el espacio europeo de educación. España: Ministerio de Educación y ciencia.

Hernández Sampieri, R., Fernández Collado, C., \& Baptista Lucio, P. (2003). Metodología de la 
investigación. México: Mc Graw Hill.

Jaik Dipp , A., \& Ortega Rocha, E. (2013). Nivel de dominio de competencias de alumnos de posgrado. En XI Congreso Nacional de Invsetigación Educativa. Durango: Centro Interdisciplinario de Investigación para el Desarrollo Integral Regional.

Marrero Sánchez , O., \& Pérez Zulueta, M. A. (2014). Competencias investigativas en la educación superior. Res Non Verba, 55-68.

Martínez, C. (2012). Estadística y Muestreo. Bogotá: Ecoe.

Muñoz Giraldo, J. F., \& Quintero Corzo, J. (2001). Competencias Investigativas para profesionales que forman y enseñan. ¿Como desarrollarlas? :. Bogotá: Editorial Magisterio.

Murcia Rodriguez, J. C. (2015). Propuesta didáctica para desarrollar competencias investigativas en estudiantes de carreras técnicas profesionales en el centro de investigación, docencia y consultoría administrativa- cidca- Bogotá. Bogotá: Universidad Militar Nueva Granada.

Nápoles Sayous, N., Beatón Soler, P., Cruz Baranda, S., \& Álvarez Gonzalez , I. (2007). La investigación científica y el aprendizaje social para la producción de conocimientos en la formación del ingeniero civil. Revista Académica Ingenieria, 11(2), 39-36.

Ochoa, M. L. (2013). Lineamientis para la seleccion y evaluacion de docentes y estudiantes y el desarrollo docente. Bogotá: Secab Publicaciones.

Pimienta Prieto, J. H. (2012). Las competencias en la docencia universitaria. México: Pearson.

Quinn, R. E., Faerman, S. R., Thompson, M. P., \& McGrath, M. R. (1994). Maestría en la Gestión de Organizaciones: Un modelo operativo de Competencias (Vol. 1). Madrid: Diaz Santos.

Rodríguez Jiménez, Y. J. (2009). La formación de docentes investigadores: lineamientos pedagógicos para su inserción en los currículos. Teoria y praxis investigativa, 4(1), 25-32.

Salas Zapata, W. A. (2002). Formación por competencias en Educación Superior: Una aproximación conceptual a propósito del caso colombiano. Revista Iberoamericana, 36.

Salazar Botello, C. M., Chiang Vega, M. M., \& Muñoz Jara, Y. A. (2016). Competencias docentes en la educacion superior: Un estudio Empirico en la universidad del Bio- Bío. Revista electronica Actividades investigativas en Educación, 16(1), 1-28.

Segura Bazán, M. (2004). Hacia un perfil del docente Universitario. Ciencias de la Educación, 1(23), 9-28.

Tobon, S. (2006). Aspectos básicos de la formación basada en competencias. . Talca: Proyecto Mesesup. 